\section{EL CÁNCER COLORRECTAL EN LA MUJER}

\author{
Rocío García-Carbonero \\ Hospital Universitario Doce de Octubre \\ rgcarbonero@gmail.com
}

\section{COLORECTAL CANCER AMONG WOMEN}

Copyright: () 2015 CSIC. Este es un artículo de acceso abierto distribuido bajo los términos de la licencia Creative Commons Attribution-Non Commercial (by-nc) Spain 3.0.
RESUMEN: El cáncer colorrectal (CCR) es el tercer tumor más frecuente en el mundo. Los datos epidemiológicos demuestran que tanto la incidencia como la mortalidad debidos a este tumor son menores en las mujeres que en los hombres. De hecho, numerosos estudios observacionales y experimentales sugieren que los estrógenos, tanto endógenos como exógenos, podrían jugar un papel protector relevante. En este artículo se van a discutir las diferencias epidemiológicas y sociológicas del CCR en función del género, algunas peculiaridades y barreras específicas para la prevención y el diagnóstico precoz del CCR en la mujer, y aspectos particulares de género sobre el impacto de las diversas terapias antineoplásicas, como son cirugía, radioterapia y quimioterapia, en la función sexual, la continencia esfinteriana y la calidad de vida de las mujeres.

PALABRAS CLAVE: calidad de vida; diagnóstico; estrógenos; prevención.
ABSTRACT: Colorectal cancer (CRC) is the third most common tumour in the world. Epidemiological data demonstrate that both the incidence and mortality of CRC is lower in women than in men. In fact, several observational and experimental studies suggest that oestrogens, both endogenous and exogenous, could play a relevant protective role. In this article, we discuss the epidemiological and sociological differences regarding gender in CRC, some particularities and specific barriers for the prevention and early diagnosis of CRC in women, as well as particular aspects of gender regarding the impact of the various antineoplastic therapies, such as surgery, radiotherapy and chemotherapy, sexual function, sphincter continence and quality of life in women.

KEYWORDS: diagnosis; estrogens; prevention; quality of life. 


\section{INTRODUCCIÓN}

El cáncer colorectal (CCR) es el tercer tumor más frecuente en el mundo, con 1.361 .000 de casos nuevos y 694.000 defunciones registradas en 2012 (http://globocan.iarc.fr). Los datos epidemiológicos demuestran que tanto la incidencia como la mortalidad son menores en las mujeres que en los hombres (De Angelis et al., 2014). Las causas de estas diferencias de género no están claras, pero numerosos estudios observacionales y experimentales sugieren que los estrógenos, tanto endógenos como exógenos, podrían jugar un papel protector relevante. El cribado poblacional del CCR a partir de los 50 años es coste-eficaz, ya que el CCR es una enfermedad frecuente y asociada a una morbi-mortalidad importante, tiene una historia natural conocida con un periodo de transición adenoma-carcinoma en general prolongado, y su detección precoz y tratamiento en estadíos iniciales mejora sustancialmente el pronóstico de la enfermedad. La adherencia a los programas de cribado, no obstante, es mucho menor que la que se ha conseguido en otros programas de prevención (p.ej. cáncer de mama), y es particularmente baja en mujeres. En este capítulo se van a discutir las diferencias epidemiológicas y sociológicas del CCR en función del género, algunas peculiaridades y barreras específicas para la prevención y el diagnóstico precoz del CCR en la mujer, y algunos aspectos particulares de género sobre el impacto de las diversas terapias antineoplásicas (cirugía, radioterapia y quimioterapia) en la función sexual, la continencia esfinteriana y la calidad de vida de las mujeres.

\section{EPIDEMIOLOGÍA DEL CÁNCER COLORRECTAL: DI- FERENCIAS DE GÉNERO}

EL CCR es una enfermedad neoplásica de una gran relevancia sanitaria. La incidencia del CCR es notablemente superior en hombres que en mujeres, con un número de nuevos casos diagnosticados por 100.000 habitantes y año de 37,3 versus 23,6 en la Unión Europea, y de 43,9 versus 24,2 en España, respectivamente (http://globocan.iarc.fr). Una tendencia similar de género se observa en las tasas de mortalidad, siendo el número de defunciones/100.000 habitantes y año de 16,2 en varones frente a 9,9 en mujeres en la Unión Europea, y de 17,1 y 8,4 en nuestro país, respectivamente (De Angelis et al., 2014) (Tabla 1). En muchos países de nuestro entorno (EEUU, Dinamarca, Francia, Reino Unido), la mortalidad por CCR ha decrecido paulatinamente en las últimas décadas, y en algunos de ellos (EEUU, Francia) ha disminuido también la incidencia, posiblemente debido en parte a la implantación con éxito de programas de cribado poblacional y a la resección endoscópica precoz de pólipos colónicos premalignos. Curiosamente, la magnitud del descenso de la mortalidad observado en estos países ha sido mayor en mujeres que en hombres. Lamentablemente es el caso de nuestro país, donde todas estas cifras continúan en ascenso.

A pesar de los incuestionables avances diagnósticos y terapéuticos alcanzados (Casado-Saenz et al., 2013), incluyendo la implantación paulatina, con más o menos éxito, de los programas de cribado poblacional en distintos países, más del $40 \%$ de los pacientes fallecen

Tabla 1. Incidencia y mortalidad del cáncer colorrectal

\begin{tabular}{|c|c|c|c|c|c|c|c|c|}
\hline & \multicolumn{2}{|c|}{ Global } & \multicolumn{2}{|c|}{ EEUU } & \multicolumn{2}{|c|}{ Europa } & \multicolumn{2}{|c|}{ España** } \\
\hline & ASRX10 5 & $\% *$ & ASRX10 6 & \%* & ASRX10 6 & $\% *$ & ASRX10 ${ }^{6}$ & $\% *$ \\
\hline \multicolumn{9}{|l|}{ Incidencia } \\
\hline Ambos sexos & 17,2 & 9,7 & 25,0 & 8,4 & 29,5 & 13,0 & 33,1 & 15,0 \\
\hline Hombres & 20,6 & 10,0 & 28,5 & 8,4 & 37,3 & 13,2 & 43,9 & 15,0 \\
\hline Mujeres & 14,3 & 9,2 & 22,0 & 8,4 & 23,6 & 12,7 & 24,2 & 14,9 \\
\hline \multicolumn{9}{|l|}{ Mortalidad } \\
\hline Ambos sexos & 8,4 & 8,5 & 9,2 & 9,0 & 12,5 & 12,2 & 12,3 & 14,3 \\
\hline Hombres & 10,0 & 8,0 & 11,0 & 8,9 & 16,2 & 11,6 & 17,1 & 13,7 \\
\hline Mujeres & 6,9 & 9,0 & 7,7 & 9,0 & 9,9 & 13,0 & 8,4 & 15,2 \\
\hline
\end{tabular}

ASR $\times 10^{5}$ : age-standardized rate $\times 100.000$ habitantes (tasas ajustadas a una distribución de edad poblacional estándar)

* \% del total de nuevos casos diagnosticados o fallecidos anualmente

** Solo el $17 \%$ de la población española con cáncer está registrada (lo datos no provienen de registros poblacionales) 
a los 5 años del diagnóstico. No obstante, la evolución temporal de las tasas de supervivencia ha sido discretamente positiva, con un incremento del porcentaje de pacientes vivos a 5 años de un 4-5\% en la última década (Tabla 2). El pronóstico del cáncer de colon y recto en nuestro país es similar a la media europea, con un índice de supervivencia a 5 años del $57.1 \%$ y $56.4 \%$, respectivamente. La supervivencia es, para ambos casos, discretamente superior en el caso de las mujeres (Tabla 3).

\section{FACTORES DE RIESGO RELACIONADOS CON EL CÁNCER COLORRECTAL EN LA MUJER}

El riesgo de padecer CCR, como en muchas otras neoplasias, aumenta con la edad, siendo la mediana de edad al diagnóstico de 72 años. Se estima que las mujeres alcanzan un nivel de riesgo de padecer CCR similar al de los hombres entre 4 y 8 años más tarde (Brenner et al., 2007; McCashland et al., 2001). Numerosos estudios observacionales, epidemiológicos y experimentales sugieren que los estrógenos podrían ejercer un efecto protector y justificar, al menos parcialmente, estas diferencias de género (Chlebowski et al., 2004; Grodstein, Newcomb y Stampfer, 1999; Manson et al., 2013; Rossouw et al., 2002; Woodson et al., 2001).

Algunos autores han atribuido el mayor descenso de mortalidad observado en mujeres desde 1990 en algunos países (p.ej. EEUU), comparado con el que el que se ha documentado en varones, al uso extendido de la terapia hormonal sustitutiva a partir de la menopausia. De hecho, un meta-análisis de 18 estudios epidemiológicos, que evaluaron la asociación entre la terapia hormonal y el riesgo de CCR en mujeres postmenopáusicas, mostró que el riesgo de padecer CCR fue un $20 \%$ menor en aquellas mujeres que habían recibido terapia hormonal sustitutiva en algún momento de su vida, y la reducción fue del 34\% para aquellas que estaban recibiendo dicho tratamiento en el momento del estudio, comparado con las mujeres que nunca tomaron hormonas (Grodstein, Newcomb y Stampfer, 1999).

En uno de los mayores ensayos clínicos de prevención primaria llevados a cabo por la Women's Health Initiative, que incluyó cerca de 17.000 mujeres postmenopáusicas (50-79 años), se observaron resultados similares. En este estudio las mujeres fueron aleatorizadas a recibir una combinación de estrógenos y acetato de medroxiprogesterona versus placebo (Chlebowski et al., 2004; Rossouw et al., 2002). Tras un seguimiento medio de 5,6 años, la incidencia de CCR fue significativamente menor en las mujeres que recibieron tratamiento hormonal combinado frente al de aquellas que recibieron placebo (HR: $0,72, p=0,014$ ), aunque conviene aclarar que este no era el objetivo primario del ensayo.

Tabla 2. Pronóstico del cáncer colorrectal en Europa. Evolución temporal

\begin{tabular}{lcc}
\hline & \multicolumn{2}{c}{ Supervivencia a 5 años (\%) } \\
\hline & Cáncer de colon & Cáncer de recto \\
\hline $1999-2001$ & 54,2 & 52,1 \\
\hline $2002-2004$ & 55,8 & 54,2 \\
\hline $2005-2007$ & 58,1 & 57,6 \\
\hline
\end{tabular}

Tabla 3. Pronóstico del cáncer colorrectal. Diferencias de género

\begin{tabular}{lcccc}
\hline \multicolumn{5}{c}{ Supervivencia a 5 años (\%) } \\
\hline Colon & Global & Hombres & Mujeres & Diferencia absoluta (p) \\
\hline Europa & & & & $1,4(<0,0001)$ \\
\hline España & 57,0 & 56,4 & 57,8 & - \\
\hline Recto & 57,1 & - & - & $2,4(0,0001)$ \\
\hline Europa & & & 57,3 & - \\
\hline España & 55,8 & 54,9 & - & \\
\hline
\end{tabular}


Esta reducción del riesgo no se documentó, por el contrario, en otro ensayo paralelo que evaluó el tratamiento con estrógenos en monoterapia (sin progestágenos) frente a placebo en más de 10.000 mujeres postmenopáusicas sin útero por cirugía previa, sí se hizo (Manson et al., 2013). Además, los cánceres detectados en las mujeres tratadas con estrógenos y progesterona en el primer estudio mencionado eran de peor pronóstico (más frecuentemente presentaban metástasis linfáticas locorregionales o a distancia) (Chlebowski et al., 2004), y por otro lado el tratamiento hormonal aumentó el riesgo cardiovascular (HR: 1,29) y la incidencia de cáncer de mama (HR: 1,26) en estas mujeres (Rossouw et al., 2002) . Globalmente, por lo tanto, se concluyó que los riesgos para la salud de esta intervención terapéutica eran mayores que los beneficios que aportaba, desaconsejándose el tratamiento hormonal como estrategia de prevención primaria, aunque su empleo puede ser considerado para el tratamiento de los síntomas menopáusicos en determinadas pacientes. Este ensayo, en cualquier caso, documenta de una manera sólida el papel protector de los estrógenos, al menos en combinación con progesterona, frente al CCR.

Los factores dietéticos también han sido estudiados con gran interés como factores de riesgo potencialmente modificables. Específicamente en mujeres, diversos estudios de cohortes (Nurses' Health Study, Health Professionals Follow-Up Study) y de intervención, incluido un ensayo clínico aleatorizado del Women's Health Initiative que incluyó más de 48.000 mujeres postmenopáusicas, no demostraron ninguna asociación entre una dieta rica en fibra y un menor riesgo de CCR (Fuchs et al., 1999; Michels et al., 2005). Tampoco se observó ningún efecto protector de una dieta pobre en grasas (Beresford et al., 2006). Por el contrario, un consumo prolongado de carne roja y procesada sí incrementa significativamente el riesgo de padecer CCR (RR 1.41 y 1.33, respectivamente) (Chao et al., 2005).

La obesidad es también un factor predisponente estrechamente relacionado con el riesgo de padecer cáncer en general y CCR en particular (Giovannucci et al., 1996; Pischon et al., 2006). En el estudio de Framingham se observó que las personas con un índice de masa corporal (IMC) $>30 \mathrm{~kg} / \mathrm{m}^{2}$ tienen un incremento del riesgo de padecer CCR del $50 \%$ para edades comprendidas entre los 30 y 54 años, y 2,4 veces superior en individuos de 55-79 años, y esta asociación fue mayor en hombres que en mujeres (Moore et al., 2004).
Estos resultados fueron confirmados en el European Prospective Investigation into Cancer and Nutrition Study (estudio EPIC), que demostró una asociación significativa entre el perímetro abdominal (indicador de obesidad central) y el riesgo de CCR tanto en hombres como en mujeres, aunque la relación con el IMC únicamente se documentó en los varones (Pischon et al., 2006). Se ha postulado que la hiperinsulinemia y la resistencia periférica a la insulina es el común denominador implicado en la promoción del desarrollo del CCR por diversos factores de riesgo ambientales como la obesidad, el aumento del perímetro abdominal, la vida sedentaria, o el consumo de un exceso de carne roja o de grasas saturadas. La implicación de esta ruta en la patogénesis del CCR ha sido demostrada en diversos modelos experimentales (Lopez-Calderero, Sanchez Chavez y Garcia-Carbonero, 2010). La implicación de otros factores como el tabaco o el alcohol es menor que en otras patologías neoplásicas y los datos de la literatura más controvertidos (Cho et al., 2004; Liang, Chen y Giovannucci, 2009).

La incidencia del CCR también varía en función de la raza, siendo el registro poblacional americano (SEER) el que mejor ha documentado este hecho. En él se ha observado una mayor incidencia de CCR en mujeres afro-americanas, seguido por orden decreciente de las mujeres de raza blanca (caucásica), indias nativas americanas y latinas/hispanas. Los factores socioeconómicos y culturales, con frecuencia asociados a la raza, también tienen una influencia importante en el acceso a una atención sanitaria de calidad y en la adherencia a los programas de cribado, como se discutirá más adelante.

Finalmente, mujeres con determinadas neoplasias ginecológicas previas tienen un mayor riesgo de padecer CCR, sobre todo si fueron diagnosticadas a edades tempranas. Por ejemplo, el registro SEER americano documentó un RR de CCR de 3,39 y 3,67 en mujeres que habían padecido un cáncer de endometrio o de ovario, respectivamente, antes de los 50 años (Weinberg, Newschaffer y Topham, 1999). Probablemente ello sea debido a que este tipo de neoplasias forman parte del síndrome de Lynch (CCR familiar no polipósico), uno de los síndromes de predisposición hereditaria al CCR más frecuente. Si todas las pacientes con carcinoma de endometrio o de ovario diagnosticadas tempranamente deben someterse a programas de cribado más precoz, o más frecuentemente, es motivo de debate. Por el contrario, otro tipo de neoplasias de la mujer como el cáncer de mama o de cérvix no parecen predisponer a un mayor riesgo de CCR (Newschaffer et al., 2001). 


\section{ESTRATEGIAS DE CRIBADO Y DIAGNÓSTICO PRE- COZ DEL CÁNCER COLORRECTAL: PECULIARIDADES Y BARRERAS ESPECÍFICAS DE GÉNERO}

Todas las estrategias de cribado muestran una mayor prevalencia de pólipos y de tumores malignos colorrectales en hombres que en mujeres, aunque estas diferencias tienden a igualarse a edades avanzadas. A pesar de ello, la edad recomendada en el momento actual para iniciar los programas de cribado poblacional (50 años en individuos sin historia familiar) no difiere en función del género (Brenner, Stock y Hoffmeister, 2014; Stracci, Zorzi y Grazzini, 2014). La colonoscopia es el método diagnóstico fundamental del CCR. Diversos estudios han demostrado que su realización es técnicamente más difícil en mujeres que en hombres por varios motivos (Church, 1994; Saunders et al., 1996). El primero de ellos es que la longitud del colon es mayor en mujeres (unos $10 \mathrm{~cm}$ más en promedio), y esto implica mayor probabilidad de bucles del intestino, mayor tiempo de exploración, mayor insuflación de aire intra-colónico, mayor dolor y requerimientos de sedación durante el procedimiento, y mayor riesgo de complicaciones (Hanson et al., 2007; Khashab et al., 2009). Otra dificultad específica de las mujeres que añade complejidad técnica a la realización de la colonoscopia es la existencia de una histerectomía u otra intervención quirúrgica pélvica previa, por las adherencias intestinales que se generan tras la cirugía (Adams et al., 2003; Ramakrishnan y Scheid, 2003; Takashi, Yanaka y Kinjo, 2005). Se sabe que en pacientes histerectomizadas la colonoscopia es más dolorosa, requiere más tiempo de exploración y más sedación, y con más frecuencia es incompleta. Estas dificultades técnicas pueden ser parcialmente solventadas empleando endoscopios flexibles, de menor calibre o colonoscopios de doble balón.

La adherencia a los programas de cribado poblacional, a pesar del beneficio demostrado, es muy baja, particularmente en mujeres y razas no caucásicas (Breen et al., 2001; Herold et al., 1997; McMahon et al., 1999; McQueen et al., 2006; Rosen y Schneider, 2004). La principal causa de no adherencia es lo poco consciente que es la población, incluidos muchos profesionales sanitarios, de que el CCR es un problema sanitario prevenible de gran relevancia. El nivel educativo y económico, en lo que se refiere al acceso a una información y atención médica adecuadas, están íntimamente relacionadas con este problema (Meissner et al., 2006; Seeff et al., 2004). Ambos son con mayor frecuencia deficientes en mujeres y en razas no caucásicas o minorías étnicas. Las mujeres son también más reacias a realizarse una colonoscopia porque lo encuentran embarazoso, y de hecho un estudio de EEUU ha demostrado que el $48 \%$ de las mujeres prefieren que les realice la colonoscopia una mujer, mientras que al $96 \%$ de los hombres les era indiferente el sexo del endoscopista (Fidler et al., 2000; Menees et al., 2005; Schneider et al., 2009; Varadarajulu, Petruff y Ramsey, 2002; Zapatier et al., 2011). No obstante, únicamente el $5 \%$ de las mujeres no se realizaron la colonoscopia por este motivo (Denberg et al., 2010) . Los antecedentes de abuso físico o emocional favorecían esta preferencia de género.

\section{IMPACTO DEL CÁNCER COLORRECTAL EN LA FUN- CIÓN SEXUAL, CONTINENCIA ESFINTERIANA Y CALI- DAD DE VIDA GLOBAL DE LAS MUJERES}

Un tercio de los cánceres colorrectales se localizan en el recto. La morbilidad de los tratamientos del cáncer de recto difiere sustancialmente de la del cáncer de colon debido a su localización pélvica. En primer lugar, la cirugía de los cánceres cercanos al esfínter anal requiere la amputación anal y la creación de un estoma permanente, lo cual condiciona de manera considerable las relaciones sociales y afectivas de los pacientes. Por otro lado, tanto la cirugía como la radioterapia pélvica pueden dañar nervios esenciales para la función sexual y para la continencia urinaria y fecal.

La disfunción sexual inducida por el tratamiento del cáncer de recto ha sido bien documentada en los varones, pero es un aspecto poco estudiado en la mujer. Aunque aproximadamente un tercio de las mujeres entre 50 y 70 años refieren ausencia de deseo sexual en condiciones normales, se estima que más de la mitad de las mujeres casadas mayores de 70 años continúan siendo sexualmente activas y más del $75 \%$ de ellas considera el sexo un aspecto importante de su vida personal (Avis et al., 2009; Beckman et al., 2008; Tan et al., 2009). La función sexual en las mujeres que han sido tratadas de un cáncer de recto puede verse afectada por múltiples causas, incluidas la pobre imagen corporal, la depresión, la pérdida de independencia, el daño de los nervios pélvicos del sistema autónomo por la cirugía o por la radioterapia, o el fallo hormonal ovárico inducido por la quimioterapia o la radioterapia (Bachmann y Leiblum, 2004; Donovan, Thompson y Hoffe, 2010; Grigsby et al., 1995; Havenga et al., 2000; Lange et al., 2009; Maas et al., 1998; Maurer et al., 2001; Ogilvy-Stuart y Shalet, 1993). La radioterapia puede además inducir atrofia y sequedad de mucosas, incluida la vaginal, fibrosis y adhesiones que en último término dificultan las relaciones sexua- 
les o las hacen dolorosas. Algunos estudios pequeños han documentado que más del $60 \%$ de las mujeres experimentan un deterioro de su función sexual tras el tratamiento del cáncer de recto, fundamentalmente por la presencia de una ostomía, así como la falta de lubricación vaginal y dispareunia (Hendren et al., 2005; Lange et al., 2009; Tekkis et al., 2009). Son, por lo tanto, secuelas permanentes frecuentes del tratamiento del CCR con un gran impacto en la calidad de vida de las pacientes, que deberían ser estudiadas con mayor profundidad.

La incontinencia urinaria es también un problema común en la mujer, con una prevalencia estimada en la comunidad de un 20-40\% según las series (Botlero et al., 2011; Botlero et al., 2009). La integridad de los nervios del sistema simpático es esencial para regular esta función, y estos se ven con frecuencia dañados como consecuencia de la cirugía y la radioterapia pélvicas. Es frecuente el aumento del tono muscular vesical, lo cual reduce la capacidad de la vejiga y favorece la incontinencia. En un estudio llevado a cabo en más de 400 pacientes con cáncer de recto (171 mujeres), el $53 \%$ presentaba incontinencia urinaria a los 5 años de haberse practicado una excisión mesorectal total, la técnica quirúrgica de elección en este tipo de neoplasias (Lange et al., 2008). La ausencia de datos basales en esta población de pacientes de este problema común hace difícil estimar con precisión en qué medida esta condición es una secuela del tratamiento. La incontinencia fecal es otro problema que afecta hasta al $50 \%$ de los pacientes con función esfinteriana normal antes del tratamiento (Peeters et al., 2005). En un estudio que aleatorizó a los pacientes con cáncer de recto a recibir o no radioterapia preoperatoria se vió que la incidencia de incontinencia fecal fue mayor en los pacientes irradiados (radioterapia y cirugía: 61\%; cirugía solo: 38\%) (Lange et al., 2007). Las diferencias en cuanto al impacto por género de este problema no se han documentado específicamente. Por todo ello, sería recomendable diseñar estudios, adecuadamente controlados, que incluyan poblaciones mayores de pacientes con seguimiento a largo plazo, para poder entender mejor la dimensión y los condicionantes de estos problemas, tan relevantes para los pacientes, y diseñar estrategias específicas de género para su adecuado manejo y seguimiento.

\section{BIBLIOGRAFÍA}

Adams, C., Cardwell, C., Cook, C., Edwards, R., Atkin, W. S. y Morton, D. G. (2003). Effect of hysterectomy status on polyp detection rates at screening flexible sigmoidoscopy. Gastrointestinal Endoscopy, 57, 7, pp. 848-853. DOI: 10.1067/ mge.2003.231S001651070300347X [pii].

Avis, N. E., Brockwell, S., Randolph, J. F., Jr., Shen, S., Cain, V. S., Ory, M. et al. (2009). Longitudinal changes in sexual functioning as women transition through menopause: results from the Study of Women's Health Across the Nation. Menopause, 16, 3, pp. 442-452. DOI: 10.1097/gme.0b013e3181948dd0.

Bachmann, G. A. y Leiblum, S. R. (2004). The impact of hormones on menopausal sexuality: a literature review. Menopause, 11, 1, pp. 120-130. DOI: 10.1097/01. GME.0000075502.60230.2800042192200411010-00020 [pii].

Beckman, N., Waern, M., Gustafson, D. y Skoog, I. (2008). Secular trends in self reported sexual activity and satisfaction in Swedish 70 year olds: cross sectional survey of four populations, 1971-2001. British Medical Journal, 337, pp. a279.
Beresford, S. A., Johnson, K. C., Ritenbaugh, C., Lasser, N. L., Snetselaar, L. G., Black, H. R. et al. (2006). Low-fat dietary pattern and risk of colorectal cancer: the Women's Health Initiative Randomized Controlled Dietary Modification Trial. Journal of the American Association, 295, 6, pp. 643-654. DOI: 295/6/643 [pii]10.1001/jama.295.6.643.

Botlero, R., Bell, R. J., Urquhart, D. M. y Davis, S. R. (2011). Prevalence of fecal incontinence and its relationship with urinary incontinence in women living in the community. Menopause, 18, 6, pp. 685-689. DOI: 10.1097/ gme.0b013e3181fee03b.

Botlero, R., Davis, S. R., Urquhart, D. M., Shortreed, S. y Bell, R. J. (2009). Agespecific prevalence of, and factors associated with, different types of urinary incontinence in community-dwelling Australian women assessed with a validated questionnaire. Maturitas, 62, 2, pp. 134-139. DOI: S0378-5122(08)004027 [pii]10.1016/j.maturitas.2008.12.017.

Breen, N., Wagener, D. K., Brown, M. L., Davis, W. W. y Ballard-Barbash, R. (2001).
Progress in cancer screening over a decade: results of cancer screening from the 1987, 1992, and 1998 National Health Interview Surveys. Journal of the National Cancer Institute, 93, 22, pp. 1704-1713.

Brenner, H., Hoffmeister, M., Arndt, V. y Haug, U. (2007). Gender differences in colorectal cancer: implications for age at initiation of screening. British Journal of Cancer, 96, 5, pp. 828-831. DOI: 6603628 [pii]10.1038/sj.bjc.6603628.

Brenner, H., Stock, C. y Hoffmeister, M. (2014). Effect of screening sigmoidoscopy and screening colonoscopy on colorectal cancer incidence and mortality: systematic review and meta-analysis of randomised controlled trials and observational studies. British Medical Journal, 348, pp. g2467.

Casado-Saenz, E., Feliu, J., Gomez-Espana, M. A., Sanchez-Gastaldo, A. y GarciaCarbonero, R. (2013). SEOM clinical guidelines for the treatment of advanced colorectal cancer 2013. Clinical and Translational Oncology, 15, 12, pp. 9961003. DOI: 10.1007/s12094-013-1082-5. 
Chao, A., Thun, M. J., Connell, C. J., McCullough, M. L., Jacobs, E. J., Flanders, W. D. et al. (2005). Meat consumption and risk of colorectal cancer. Journal of the American Association, 293, 2, pp. 172-182. DOI: 293/2/172 [pii]10.1001/ jama.293.2.172.

Chlebowski, R. T., Wactawski-Wende, J., Ritenbaugh, C., Hubbell, F. A., Ascensao, J., Rodabough, R. J. et al. (2004). Estrogen plus progestin and colorectal cancer in postmenopausal women. New England Journal of Medicine, 350, 10, pp. 991-1004. DOI: 10.1056/NEJMoa032071350/10/991 [pii].

Cho, E., Smith-Warner, S. A., Ritz, J., van den Brandt, P. A., Colditz, G. A., Folsom, A. R. et al. (2004). Alcohol intake and colorectal cancer: a pooled analysis of 8 cohort studies. Annals of Internal Medicine, 140, 8, pp. 603-613. DOI: 140/8/603 [pii].

Church, J. M. (1994). Complete colonoscopy: how often? And if not, why not? American Journal of Gastroenterology, 89, 4, pp. 556-560.

De Angelis, R., Sant, M., Coleman, M. P., Francisci, S., Baili, P., Pierannunzio, D. et al. (2014). Cancer survival in Europe 1999-2007 by country and age: results of EUROCARE--5-a population-based study. Lancet Oncology, 15, 1, pp. 23-34. DOI: S1470-2045(13)70546-1 [pii]10.1016/S1470-2045(13)70546-1.

Denberg, T. D., Kraus, H., Soenksen, A., Mizrahi, T., Shields, L. y Lin, C. T. (2010). Rates of screening colonoscopy are not increased when women are offered a female endoscopist in a health promotion outreach program. Gastrointestinal Endoscopy, 72, 5, pp. 1014-1019. DOI: S0016-5107(10)01747-5 [pii]10.1016/j. gie.2010.06.014.

Donovan, K. A., Thompson, L. M. y Hoffe, S. E. (2010). Sexual function in colorectal cancer survivors. Cancer Control, 17, 1, pp. 44-51.

Fidler, H., Hartnett, A., Cheng Man, K., Derbyshire, I. y Sheil, M. (2000). Sex and familiarity of colonoscopists: patient preferences. Endoscopy, 32, 6, pp. 481482. DOI: $10.1055 / \mathrm{s}-2000-645$.

Fuchs, C. S., Giovannucci, E. L., Colditz, G. A., Hunter, D. J., Stampfer, M. J., Rosner, B. et al. (1999). Dietary fiber and the risk of colorectal cancer and adenoma in women. New England Journal of Medicine, 340, 3, pp. 169-176. DOI: 10.1056/NEJM199901213400301.
Giovannucci, E., Colditz, G. A., Stampfer, M. J. y Willett, W. C. (1996). Physical activity, obesity, and risk of colorectal adenoma in women (United States). Cancer Causes Control, 7, 2, pp. 253-263.

Grigsby, P. W., Russell, A., Bruner, D., Eifel, P., Koh, W. J., Spanos, W. et al. (1995). Late injury of cancer therapy on the female reproductive tract. International Journal of Radiation Oncology, Biology, Physics, 31, 5, pp. 1281-1299. DOI: 03603016(94)00426-L [pii]10.1016/03603016(94)00426-L.

Grodstein, F., Newcomb, P. A. y Stampfer, M. J. (1999). Postmenopausal hormone therapy and the risk of colorectal cancer: a review and meta-analysis. American Journal of Medicine, 106, 5, pp. 574-582. DOI: S0002934399000637 [pii].

Hanson, M. E., Pickhardt, P. J., Kim, D. H. y Pfau, P. R. (2007). Anatomic factors predictive of incomplete colonoscopy based on findings at CT colonography. American Journal of Roentgenology, 189, 4, pp. 774-779. DOI: 189/4/774 [pii]10.2214/AJR.07.2048.

Havenga, K., Maas, C. P., DeRuiter, M. C., Welvaart, K. y Trimbos, J. B. (2000). Avoiding long-term disturbance to bladder and sexual function in pelvic surgery, particularly with rectal cancer. Seminars in Surgical Oncology, 18, 3, pp. 235-243. DOI: 10.1002/(SICI)10982388(200004/05)18:3<235::AID SSU7>3.0.CO;2-7 [pii]

Hendren, S. K., O'Connor, B. I., Liu, M., Asano, T., Cohen, Z., Swallow, C. J. et al. (2005). Prevalence of male and female sexual dysfunction is high following surgery for rectal cancer. Annals of Surgery, 242, 2, pp. 212-223. DOI: 00000658 200508000-00010 [pii].

Herold, A. H., Riker, A. I., Warner, E. A., Woodard, L. J., Brownlee, H. J., Jr., Pencev, D. et al. (1997). Evidence of gender bias in patients undergoing flexible sigmoidoscopy. Cancer Detection and Prevention, 21, 2, pp. 141-147.

Khashab, M. A., Pickhardt, P. J., Kim, D. H. y Rex, D. K. (2009). Colorectal anatomy in adults at computed tomography colonography: normal distribution and the effect of age, sex, and body mass index. Endoscopy, 41, 8, pp. 674-678. DOI: 10.1055/s-0029-1214899.

Lange, M. M., den Dulk, M., Bossema, E. R., Maas, C. P., Peeters, K. C., Rutten, H. J. et al. (2007). Risk factors for faecal incontinence after rectal cancer treatment. British Journal of Surgery, 94, 10, pp. 1278-1284. DOI: 10.1002/bjs.5819.

Lange, M. M., Maas, C. P., Marijnen, C. A., Wiggers, T., Rutten, H. J., Kranenbarg, E. K. et al. (2008). Urinary dysfunction after rectal cancer treatment is mainly caused by surgery. British Journal of Cancer, 95, 8, pp. 1020-1028. DOI: $10.1002 /$ bjs. 6126 .

Lange, M. M., Marijnen, C. A., Maas, C. P., Putter, H., Rutten, H. J., Stiggelbout, A. M. et al. (2009). Risk factors for sexual dysfunction after rectal cancer treatment. European Journal of Cancer, 45, 9, pp. 1578-1588. DOI: S0959-8049(08)01029-0 [pii]10.1016/j. ejca.2008.12.014

Liang, P. S., Chen, T. Y. y Giovannucci, E. (2009). Cigarette smoking and colorectal cancer incidence and mortality: systematic review and meta-analysis. International Journal of Cancer, 124, 10, pp. 2406-2415. DOI: 10.1002/ijc.24191.

Lopez-Calderero, I., Sanchez Chavez, E. y Garcia-Carbonero, R. (2010). The insulin-like growth factor pathway as a target for cancer therapy. Clinical and Translational Oncology, 12, 5, pp. 326338. DOI: 1275 [pii]10.1007/s12094010-0514-8

Maas, C. P., Moriya, Y., Steup, W. H., Kiebert, G. M., Kranenbarg, W. M. y van de Velde, C. J. (1998). Radical and nerve-preserving surgery for rectal cancer in The Netherlands: a prospective study on morbidity and functional outcome. British Journal of Cancer, 85,1 , pp. 92-97. DOI: 10.1046/j.13652168.1998.00530.x.

Manson, J. E., Chlebowski, R. T., Stefanick, M. L., Aragaki, A. K., Rossouw, J. E., Prentice, R. L. et al. (2013). Menopausal hormone therapy and health outcomes during the intervention and extended poststopping phases of the Women's Health Initiative randomized trials. Journal of the American Association, 310, 13, pp. 1353-1368. DOI: 1745676 [pii]10.1001/jama.2013.278040.

Maurer, C. A., Z'Graggen, K., Renzulli, P., Schilling, M. K., Netzer, P. y Buchler, M. W. (2001). Total mesorectal excision preserves male genital function compared with conventional rectal cancer surgery. British Journal of Cancer, 88, 11, pp. 1501-1505. DOI: 1904 [pii]10.1046/ j.0007-1323.2001.01904.x 
McCashland, T. M., Brand, R., Lyden, E. y de Garmo, P. (2001). Gender differences in colorectal polyps and tumors. American Journal of Gastroenterology, 96, 3, pp. 882-886. DOI: S00029270(00)02431-X [pii]10.1111/j.15720241.2001.3638_a.x.

McMahon, L. F., Jr., Wolfe, R. A., Huang, S., Tedeschi, P., Manning, W., Jr. y Edlund, M. J. (1999). Racial and gender variation in use of diagnostic colonic procedures in the Michigan Medicare population. Medical Care, 37, 7, pp. 712-717.

McQueen, A., Vernon, S. W., Meissner, H. I. Klabunde, C. N. y Rakowski, W. (2006). Are there gender differences in colorectal cancer test use prevalence and correlates? Cancer Epidemiology, Biomarkers and Prevention, 15, 4, pp. 782-791. DOI: 15/4/782 [pii]10.1158/1055-9965. EPI-05-0629.

Meissner, H. I., Breen, N., Klabunde, C. N. y Vernon, S. W. (2006). Patterns of colorectal cancer screening uptake among men and women in the United States. Cancer Epidemiology, Biomarkers and Prevention, 15, 2, pp. 389-394. DOI: 15/2/389 [pii]10.1158/1055-9965. EPI-05-0678.

Menees, S. B., Inadomi, J. M., Korsnes, S. y Elta, G. H. (2005). Women patients' preference for women physicians is a barrier to colon cancer screening. Gastrointestinal Endoscopy, 62, 2, pp. 219223. DOI: S0016510705005407 [pii].

Michels, K. B., Fuchs, C. S., Giovannucci, E., Colditz, G. A., Hunter, D. J., Stampfer, M. J. et al. (2005). Fiber intake and incidence of colorectal cancer among 76,947 women and 47,279 men. Cancer Epidemiology, Biomarkers and Prevention, 14 , 4, pp. 842-849. DOI: 14/4/842 [pii]10.1158/1055-9965.EPI-04-0544.

Moore, L. L., Bradlee, M. L., Singer, M. R., Splansky, G. L., Proctor, M. H., Ellison, R. C. et al. (2004). BMI and waist circumference as predictors of lifetime colon cancer risk in Framingham Study adults. International Journal of Obesity and Related Metabolic Disorders, 28, 4, pp. 559-567. DOI: 10.1038/ sj.ijo.08026060802606 [pii].

Newschaffer, C. J., Topham, A., Herzberg, T., Weiner, S. y Weinberg, D. S. (2001). Risk of colorectal cancer after breast cancer. Lancet, 357, 9259, pp. 837-840. DOI: S0140-6736(00)04197-0 [pii]10.1016/ S0140-6736(00)04197-0.
Ogilvy-Stuart, A. L. y Shalet, S. M. (1993). Effect of radiation on the human reproductive system. Environmental Health Perspectives, 101 Suppl 2, pp. 109-116.

Peeters, K. C., van de Velde, C. J., Leer, J. W., Martijn, H., Junggeburt, J. M., Kranenbarg, E. K. et al. (2005). Late side effects of short-course preoperative radiotherapy combined with total mesorectal excision for rectal cancer: increased bowel dysfunction in irradiated patients--a Dutch colorectal cancer group study. Journal of Clinical Oncology, 23, 25, pp. 6199-6206. DOI: 23/25/6199 [pii]10.1200/JCO.2005.14.779.

Pischon, T., Lahmann, P. H., Boeing, H., Friedenreich, C., Norat, T., Tjonneland, A. et al. (2006). Body size and risk of colon and rectal cancer in the European Prospective Investigation Into Cancer and Nutrition (EPIC). Journal of the National Cancer Institute, 98, 13, pp. 920 931. DOI: 98/13/920 [pii]10.1093/jnci/ djj246.

Ramakrishnan, K. y Scheid, D. C. (2003). Predictors of incomplete flexible sigmoidoscopy. Journal of the American Board of Family Practice, 16, 6, pp. 478 484. DOI: 16/6/478 [pii]

Rosen, A. B. y Schneider, E. C. (2004). Colorectal cancer screening disparities related to obesity and gender. Journal of General Internal Medicine, 19, 4, pp. 332-338. DOI: 10.1111/j.15251497.2004.30339.xJGI30339 [pii].

Rossouw, J. E., Anderson, G. L., Prentice, R. L., LaCroix, A. Z., Kooperberg, C., Stefanick, M. L. et al. (2002). Risks and benefits of estrogen plus progestin in healthy postmenopausal women: principal results From the Women's Health Initiative randomized controlled trial. Journal of the American Association, 288,3 , pp. 321-333. DOI: joc21036 [pii].

Saunders, B. P., Fukumoto, M., Halligan, S., Jobling, C., Moussa, M. E., Bartram, C. I. et al. (1996). Why is colonoscopy more difficult in women? Gastrointestinal Endoscopy, 43, 2 Pt 1, pp. 124-126. DOI: S0016510796000533 [pii].

Schneider, A., Kanagarajan, N., Anjelly, D., Reynolds, J. C. y Ahmad, A. (2009). Importance of gender, socioeconomic status, and history of abuse on patient preference for endoscopist. American Journal of Gastroenterology, 104, 2, pp. 340-348. DOI: ajg200896 [pii]10.1038/ ajg.2008.96
Seeff, L. C., Nadel, M. R., Klabunde, C. N., Thompson, T., Shapiro, J. A., Vernon, S. W. et al. (2004). Patterns and predictors of colorectal cancer test use in the adult U.S. population. Cancer, 100, 10, pp. 2093-2103. DOI: 10.1002/cncr.20276.

Stracci, F., Zorzi, M. y Grazzini, G. (2014). Colorectal cancer screening: tests, strategies, and perspectives. Frontiers in Public Health, 2, pp. 210. DOI: 10.3389/ fpubh.2014.00210.

Takashi, Y., Yanaka, H. y Kinjo, M. (2005). Prospective evaluation of factors predicting difficulty and pain during sedation-free colonoscopy. Diseases of the Colon and Rectum, 48, pp. 1295-1300.

Tan, H. M., Marumo, K., Yang, D. Y., Hwang, T. I. y Ong, M. L. (2009). Sex among Asian men and women: the Global Better Sex Survey in Asia. International Journal of Urology, 16, 5, pp. 507-514; discussion 514-505. DOI: IJU2283 [pii]10.1111/ j.1442-2042.2009.02283.x.

Tekkis, P. P., Cornish, J. A., Remzi, F. H., Tilney, H. S., Strong, S. A., Church, J. M. et al. (2009). Measuring sexual and urinary outcomes in women after rectal cancer excision. Diseases of the Colon and Rectum, 52, 1, pp. 46-54. DOI: $\quad 10.1007 /$ DCR.0b013e31819755 1e00003453-200901000-00008 [pii].

Varadarajulu, S., Petruff, C. y Ramsey, W. H. (2002). Patient preferences for gender of endoscopists. Gastrointestinal Endoscopy, 56, 2, pp. 170-173. DOI: S0016510702000342 [pii].

Weinberg, D. S., Newschaffer, C. J. y Topham, A. (1999). Risk for colorectal cancer after gynecologic cancer. Annals of Internal Medicine, 131, 3, pp. 189193. DOI: $199908030-00005$ [pii].

Woodson, K., Lanza, E., Tangrea, J. A., Albert, P. S., Slattery, M., Pinsky, J. et al. (2001). Hormone replacement therapy and colorectal adenoma recurrence among women in the Polyp Prevention Trial. Journal of the National Cancer Institute, 93, 23, pp. 1799-1805.

Zapatier, J. A., Kumar, A. R., Perez, A., Guevara, R. y Schneider, A. (2011). Preferences for ethnicity and sex of endoscopists in a Hispanic population in the United States. Gastrointestinal Endoscopy, 73, 1, pp. 89-97, 97 e81-84. DOI: S0016-5107(10)02090-0 [pii]10.1016/j. gie.2010.09.008 